\title{
Regulated polyadenylation controls mRNA translation during meiotic maturation of mouse oocytes
}

\author{
Jean-Dominique Vassalli, ${ }^{1}$ Joaquin Huarte, ${ }^{1}$ Dominique Belin, ${ }^{2}$ Pascale Gubler, ${ }^{1}$ Anne Vassalli, ${ }^{1,4}$ \\ Marcia L. O'Connell, ${ }^{3}$ Lance A. Parton, ${ }^{3}$ Richard J. Rickles, ${ }^{3}$ and Sidney Strickland ${ }^{3}$ \\ ${ }^{1}$ Institute of Histology and Embryology, and ${ }^{2}$ Department of Pathology, University of Geneva Medical School, CH 1211 \\ Geneva 4, Switzerland; ${ }^{3}$ Department of Molecular Pharmacology, State University of New York, Stony Brook, New York \\ 11794 USA
}

\begin{abstract}
The translational activation of dormant tissue-type plasminogen activator mRNA during meiotic maturation of mouse oocytes is accompanied by elongation of its $3^{\prime}$-poly(A) tract. Injected RNA fragments that correspond to part of the $3^{\prime}$-untranslated region (3'UTR) of this mRNA are also subject to regulated polyadenylation. Chimeric mRNAs containing part of this $3^{\prime}$ UTR are polyadenylated and translated following resumption of meiosis. Polyadenylation and translation of chimeric mRNAs require both specific sequences in the $3^{\prime} U T R$ and the canonical 3'-processing signal AAUAAA. Injection of 3'-blocked mRNAs and in vitro polyadenylated mRNAs shows that the presence of a long poly(A) tract is necessary and sufficient for translation. These results establish a role for regulated polyadenylation in the post-transcriptional control of gene expression.
\end{abstract}

[Key Words: Plasminogen activators; meiosis; poly(A); 3'UTR; translational control]

Received August 18, 1989; revised version accepted October 13, 1989.

A rapid and efficient mechanism to regulate gene expression involves the post-transcriptional modulation of mRNA translation or stability. There are many examples of this type of regulation (Ilan 1987), including the iron-regulated translation of ferritin mRNA (Zähringer et al. 1976; Shull and Theil 1982) and stability of transferrin receptor mRNA (Müllner and Kühn 1988 , and the regulation of $\beta$-tubulin mRNA life span by the protein product (Yen et al. 1988). A striking case of post-transcriptional control is provided by the uncoupling of gene transcription and translation in oocytes: During oocyte growth, transcription of a number of genes yields stable, untranslated mRNAs, whose translation and degradation are triggered by precisely timed developmental events (Davidson 1986).

Studies on the mechanisms controlling activation of dormant mRNAs in oocytes and fertilized eggs from different phyla have revealed a correlation between the extent of polyadenylation of a message and its translation (Colot and Rosbash 1982; Rosenthal et al. 1983; Dworkin and Dworkin-Rastl 1985; Huarte et al. 1987b; Rosenthal and Ruderman 1987; Goldman et al. 1988; Hyman and Wormington 1988; Mutter et al. 1988; Paynton et al. 1988; McGrew et al. 1989|. Extension of a pre-existing short poly $(\mathrm{A})$ tail is associated with mRNA activation, and deadenylation is correlated with the cessation of translation. However, whether these changes

4Present address: Department of Biology, Massachusetts Institute of Technology, Cambridge, Massachusetts 02139 USA. in polyadenylation trigger or merely accompany translation is not known. In fact, since its discovery 20 years ago (Edmonds and Caramela 1969; Darnell et al. 1971; Edmonds et al. 1971; Lee et al. 1971), it has not been possible to assign a definitive role to the poly(A) tract that is present at the $3^{\prime}$ end of most eukaryotic mRNAs (Brawerman 1981). Not all mRNAs are polyadenylated, indicating that this modification is not an absolute prerequisite for translation. Presence of a poly(A) tract can influence stability and/or translatability of heterologous mRNAs injected into Xenopus laevis oocytes or added to a reticulocyte lysate (Huez et al. 1974; Doel and Carey 1976; Drummond et al. 1985). However, a causal role for polyadenylation in translation or stability of an endogenous mRNA has not been demonstrated.

The mRNA for tissue-type plasminogen activator (tPA) accumulates in growing mouse oocytes, and it is stored, stable and untranslated, in the cytoplasm of the meiotically arrested primary oocyte. Following resumption of meiosis, starting $\sim 3 \mathrm{hr}$ after dissolution of the nuclear membrane (germinal vesicle breakdown, GVBD|, tPA mRNA undergoes a progressive $3^{\prime}$ polyadenylation; concomitantly, it is translated and then degraded (Huarte et al. 1985, 1987b). Thus, the extent of polyadenylation of this message is correlated with its translation and subsequent loss of stability. To examine the possible interrelationship between these phenomena, we injected into mouse oocytes various RNA fragments and chimeric mRNAs and studied their fate under conditions that prevent or allow meiotic matura- 
tion. The results demonstrate that cytoplasmic $3^{\prime}$ polyadenylation, regulated by sequences present in the $3^{\prime}$-untranslated region ( $3^{\prime} \mathrm{UTR}$ ) of tPA mRNA, is necessary and sufficient for translational activation; thus they establish a role for polyadenylation in the post-transcriptional control of gene expression.

\section{Results \\ Regulated polyadenylation of injected RNAs}

Previous experiments demonstrated that the $3^{\prime} \mathrm{UTR}$ of tPA mRNA plays a critical role in its translational activation during meiotic maturation. Injection of antisense RNAs into mouse oocytes had shown that (1) in contrast to other parts of the molecule, the $3^{\prime} U T R$ of tPA mRNA is accessible to hybrid formation in the primary oocyte; and (2) hybrid formation leads to mRNA cleavage, and amputation of the $3^{\prime}$-terminal sequences of tPA mRNA prevents its polyadenylation, translation, and degradation (Strickland et al. 1988).

Thus, it appeared that the 3'UTR might contain structural determinants that allow recognition of tPA mRNA during meiotic maturation. To investigate this possibility, oocytes were injected with a 487-nucleotide RNA fragment that corresponds to the end of the $3^{\prime} \mathrm{UTR}$ of tPA mRNA (Fig. 1A). Northern blot analysis of total RNA revealed that the fate of this fragment was similar to that of the endogenous, $2.5-\mathrm{kb}$ tPA mRNA transcript: Both molecules were essentially stable in primary oocytes and were progressively elongated and degraded in maturing oocytes (Fig. 2A). As shown previously for endogenous tPA mRNA (Huarte et al. 1987b), elongation of the injected fragment was the result of the addition of a poly(A) tract at the $3^{\prime}$ end, as treatment with RNase $\mathrm{H}$ in the presence of oligo(dT) reduced its size to that of the uninjected transcript (Fig. 2B). The efficiency of processing of this RNA fragment was remarkable: in experiments in which injected oocytes contained up to $300-$ fold more fragment than endogenous tPA mRNA, both species appeared to be completely processed. Thus, in maturing oocytes, the polyadenylation machinery is clearly not present in limiting amount.

To determine whether specific features of this 487nucleotide RNA are required for polyadenylation, other RNA fragments were injected. A fragment lacking the terminal 99 nucleotides of tPA sequence, and therefore lacking the 3'-processing signal AAUAAA (Proudfoot and Brownlee 1976), was not polyadenylated (Fig. 2C). However, not all RNAs that contained AAUAAA were elongated during meiotic maturation: A radiolabeled rabbit $\beta$-globin RNA fragment, containing the $3^{\prime}$ AAUAAA sequence of the mRNA (Fig. 1A), was coinjected with the radiolabeled 487-nucleotide tPA mRNA 3'UT fragment described above; electrophoretic analysis of the RNAs recovered after maturation demonstrated that, in contrast to the tPA-derived fragment, the $\beta$ globin fragment was not elongated (Fig. 2D). Similarly, despite the presence of an AAUAAA element close to its 3 ' extremity, a fragment containing mouse urokinase-

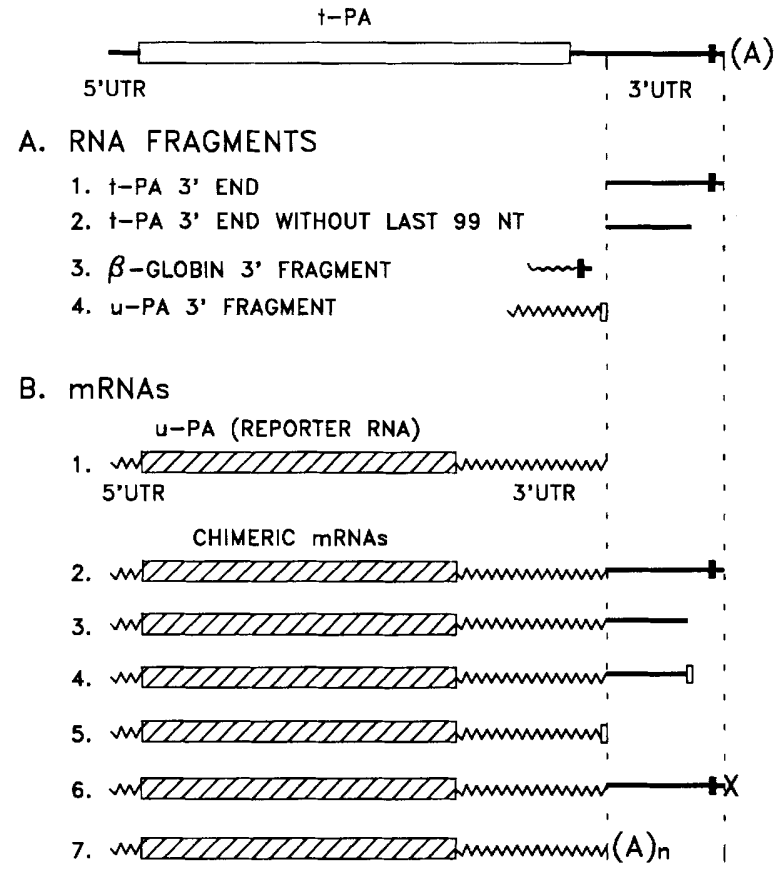

Figure 1. Schematic representation of the transcripts. The drawing is to scale; the endogenous tPA mRNA has a length of $2.5 \mathrm{~kb}$. Lines represent UTRs; rectangles represent coding regions. Small solid rectangles represent a natural AAUAAA sequence; small open rectangles represent a synthetic AAUAAA sequence. (A) Short poly(A) tail $(<40 \mathrm{As}) ;[(\mathrm{A}) \mathrm{n}]$ long poly(A) tail (200-400 As); (X) cordycepin.

type PA (uPA) mRNA sequences (Fig. 1A) was not detectably elongated in maturing oocytes (Fig. 2D). Finally, transcripts generated from the plasmid used for fragments $A(1)$ and $A(2)$ (Fig. 1), but linearized at different sites downstream from the tPA insert and, hence, containing up to 180 nucleotides of vector sequences and a different nucleotide at their 3 ' ends, were all elongated during meiotic maturation (Fig. 3). The longest fragment was found to undergo only limited polyadenylation, suggesting that the effect of tPA-derived sequences may decrease with distance from the end of the transcript. We conclude that $(1)$ the nature of the $3^{\prime}$-terminal base is irrelevant, (2) the precise distance from the tPA sequences to the $3^{\prime}$ end of the injected molecule is not critical, (3) the presence of tPA 3' sequences, including possibly AAUAAA, is essential, and (4) the AAUAAA sequence is not sufficient.

\section{Translation of chimeric mRNAs}

The results obtained with injected RNA fragments implied that a recognition process involving the 3'UTR of tPA mRNA is obligatory for polyadenylation during meiotic maturation. Thus, it seemed possible that this region contains some, and perhaps all, of the determinants for timed translational activation. To explore this issue, we prepared a chimeric mRNA, composed of a reporter mRNA joined to the last 455 nucleotides of 


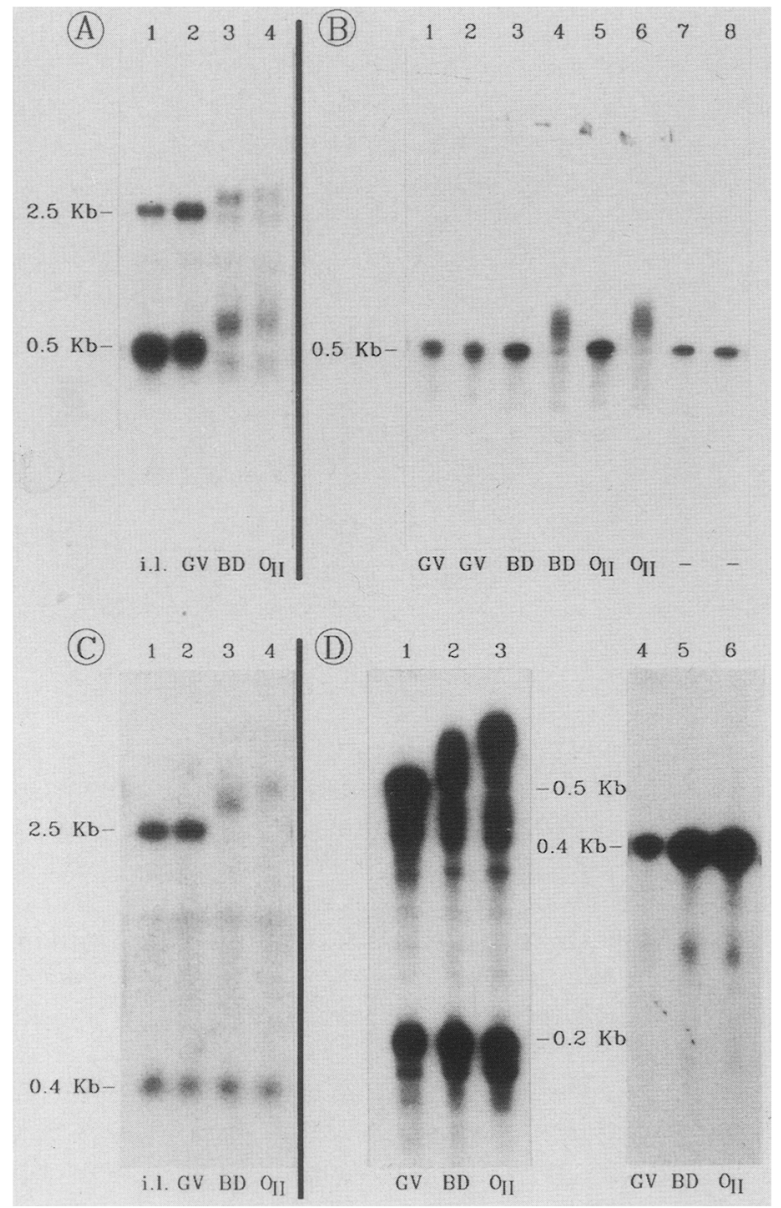

Figure 2. Polyadenylation of injected RNA fragments. $(A)$ Northern blot analysis of total RNA from oocytes injected with a 0.5-kb RNA fragment containing the end of the $3^{\prime}$ UTR of tPA mRNA [Fig. 1, fragment A(1)]. Following injection, oocytes (in pools of eight) were either lysed immediately (i.l., lane 1 ) or after culture for $10-12 \mathrm{hr}$ in the presence of dBcAMP [to prevent resumption of meiosis [GV, lane 2)], or in the absence of dBcAMP [maturing oocytes that have undergone GVBD but without polar body (BD, lane 3); secondary oocytes that have extruded the first polar body $\left(\mathrm{O}_{\mathrm{II}}\right.$ lane 4$\left.)\right]$. The probe used recognizes both the endogenous $2.5-\mathrm{kb}$ tPA mRNA and the $0.5-\mathrm{kb}$ injected fragment. $(B) \mathrm{Gel}$ analysis of radiolabeled transcripts injected into oocytes, isolated after culture, and treated with RNase $\mathrm{H}$ in the presence or absence of oligo(dT). Fragment $\mathrm{A}(\mathrm{l})$ (Fig. 1) was injected and recovered from GV, BD, and $\mathrm{O}_{\mathrm{II}}$ oocytes as for $A$, or analyzed directly (lanes 7 and 8 ). Each sample was treated with RNase $\mathrm{H}$ in the presence (lanes $1,3,5$, and 7) or absence (lanes 2, 4, 6, and 8) of oligo(dT). The RNAs were electrophoresed in a polyacrylamide/urea gel and detected by autoradiography. $(C)$. Northern blot analysis of total RNA from oocytes injected with a $0.4-\mathrm{kb}$ RNA fragment from the $3^{\prime}$ UTR of tPA mRNA, which lacks the 99 nucleotides, 3' terminal of the cDNA [Fig. 1, fragment A(2)]. Samples were prepared and analyzed as for panel $A$. The probe used recognizes both the endogenous $2.5-\mathrm{kb}$ tPA mRNA and the $0.4-\mathrm{kb}$ injected fragment. $(D)$ Gel analysis of radiolabeled transcripts after injection into oocytes. Fragments $\mathrm{A}(1)$ and $\mathrm{A}(3)$ (injected together, lanes 1-3) and fragment A(4) (injected alone, lanes 4-6) were recovered and analyzed by polyacrylamide/urea gel electrophoresis and autoradiography as for $B$.

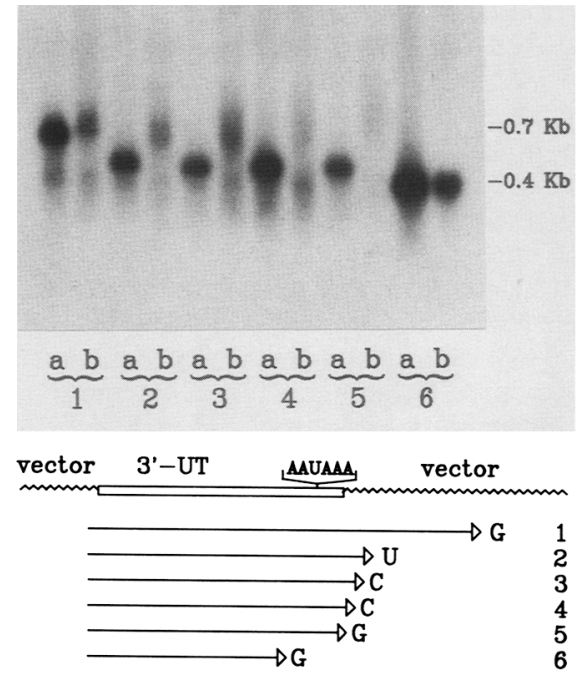

Figure 3. Polyadenylation of injected RNA fragments. The plasmid used to generate fragments $A(1)$ and $A(2)$ (Fig. 1) was also linearized with different restriction enzymes to yield transcripts with different nucleotides at their $3^{\prime}$ ends: (1) PvuII, $3^{\prime}$ terminal $G$ preceded by 180 nucleotides of vector sequences; (2) EcoRI, 3'-terminal U; (3) SmaI, 3'-terminal C; (4) BamHI, 3'-terminal C; (5) XbaI, 3'-terminal G [Fig. 1, fragment A(1)]; (6) SpeI, 3 '-terminal G [Fig. 1, fragment A(2)]. (Lanes a) Uninjected fragments $(200 \mathrm{pl}$ ); (lanes $b)$ total RNA from pools of 10 oocytes injected with $10 \mathrm{pl}$ of RNA fragments and lysed after 10-12 hr of culture in the absence of dBcAMP (maturing oocytes). The samples were prepared and analyzed by Northern blot hybridization, as for Fig. 2A.

the $3^{\prime}$ UTR of tPA mRNA (Fig. 1B). As reporter mRNA, we chose that coding for murine uPA. Mouse primary and secondary oocytes do not contain detectable uPA mRNA (data not shown) or enzymatic activity (Huarte et al. 1985). The same highly sensitive zymographic procedure (enzyme assay after electrophoresis) can be used to reveal both uPA and tPA; furthermore, the different molecular weights of the two enzymes allow them to be visualized simultaneously, so that translation of endogenous ( $\mathrm{tPA}$ ) and injected (UPA) mRNAs can be assessed in the same sample.

Oocytes were injected with the reporter uPA mRNA transcript (Fig. 1B, transcript 1) and cultured as arrested primary or as maturing oocytes. Neither sample contained detectable uPA enzyme, whereas maturing oocytes contained tPA activity, as expected (Fig. 4, lanes 1 and 2). In contrast, maturing oocytes that had been injected with a uPA/tPA chimeric transcript (Fig. 1B, transcript 2) contained both uPA and tPA; neither uPA nor tPA was synthesized if the cells had been cultured as arrested primary oocytes (Fig. 4, lanes 3 and 4). We conclude that 455 nucleotides of the $3^{\prime}$ UTR of tPA mRNA impart to the reporter mRNA the same translational control as that affecting the endogenous tPA mRNA.

The amount of uPA produced was proportional to the concentration of chimeric transcript injected; at 100 $\mathrm{ng} / \mu \mathrm{l}$, injected oocytes contained approximately equivalent catalytic amounts of UPA and tPA. This concentra- 
Figure 4. Translation of injected reporter and chimeric mRNAs. (Lanes 1-10) Transcripts corresponding to mRNAs $B(1-5)$ (Fig. 1B) were injected, the oocytes were cultured as described in Fig. $2 \mathrm{~A}$, collected in groups of three, lysed in Triton X-100, and assayed by zymography. The dark areas in the illustration represent zones of substrate lysis due to the presence of tPA $\left(M_{\mathrm{r}} 72,000\right)$ or uPA $\left(M_{\mathrm{r}} 48,000\right)$ activity in the samples; immunoprecipitation of the $M_{\mathrm{r}} 48,000$ enzyme with anti-murine uPA antibodies (Belin et al. 1984) confirmed that it is uPA (data not shown). For each mRNA, experiments using four to eight separate transcriptions were performed and a total of 13-26 pools of maturing (BD or $\mathrm{O}_{\mathrm{II}}$ ) oocytes was analyzed. (Lanes 11-16) The same transcripts were injected into stage VI $X$. laevis oocytes; oocytes were also injected with buffer alone (-, lane 11). Following $20 \mathrm{hr}$ of culture, the oocytes were analyzed by zymography.

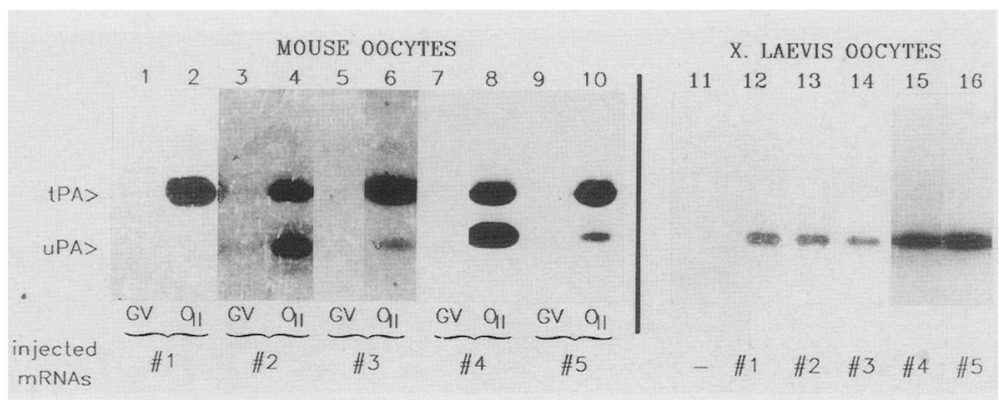

tion was selected for all subsequent experiments, as the tPA activity thereby provides an internal control to which the amount of uPA produced following injection of different chimeric transcripts can be compared. However, the translational efficiency of endogenous and injected mRNAs cannot be directly compared from our results, because the specific activities of murine UPA and tPA in the zymographic assay are not known and may be different.

From experiments using injection of an antisense transcript (Strickland et al. 1988), we knew that the 103 nucleotides, $3^{\prime}$-terminal of tPA mRNA, are necessary for its translational activation. In accord with this previous observation, a chimeric mRNA lacking this segment of the 3'UTR (Fig. 1B, transcript 3) was inefficiently translated in maturing oocytes (uPA approximately one-tenth of endogenous tPA; Fig. 4, lanes 5 and 6), confirming that determinant(s) in this region, which includes the AAUAAA signal, are required for full translational activation. To evaluate the importance of the AAUAAA sequence, this inefficiently translated mRNA was modified so as to contain an AAUAAA element near its $3^{\prime}$ end (Fig. 1B, transcript 4); this transcript was well translated in maturing oocytes (Fig 4, lanes 7 and 8). Because the only common sequence between the last $103 \mathrm{nu}$ cleotides of the endogenous mRNA and the sequences added to transcript 3 are AAUAAA, this result provides strong evidence for the need for an AAUAAA signal.
However, this signal was not sufficient, because the uPA reporter mRNA modified to contain AAUAAA near its $3^{\prime}$ end, but lacking tPA-specific sequences (Fig. 1B, transcript 5 ), yielded only low levels of uPA (approximately one-fifth of endogenous tPA; Fig. 4, lanes 9 and 10). Therefore, both the AAUAAA signal and other determinants in the 3'UTR of tPA mRNA are necessary to impart efficient translational control to the injected reporter mRNA.

The differences in translation of the injected mRNAs reflect properties specific to the translational control system of the mouse oocyte. Indeed, the five transcripts described above were translated with similar efficiency in two heterologous systems, that is, meiotically arrested stage VI $X$. laevis oocytes (uPA synthesis was evaluated by zymographic analysis of lysates of injected oocytes; Fig. 4, lanes 11-16) and wheat germ lysate (as determined by $\left[{ }^{35} \mathrm{~S} \mid\right.$ methionine labeling, SDS-PAGE, and autoradiography; data not shown).

Northern blot hybridization (Fig. 5) showed that the injected mRNAs did not differ markedly in their stability, either in primary or in maturing oocytes; the difference in their translation is thus not due to different rates of degradation. Interestingly, only the transcripts that were efficiently translated ( 2 and 4 ) were found to undergo a structural modification in maturing oocytes. As described for endogenous tPA mRNA (Huarte et al. 1987b; see also Fig. 2) and for the isolated 3'UTR frag-
Figure 5. Northern blot analysis of injected reporter and chimeric mRNAs. Oocytes were injected with the same transcripts as for Fig. 4; they were lysed either immediately (i.l.) or after culture. RNA was extracted from pools of eight oocytes and analyzed by Northern blot hybridization using a probe specific for the reporter uPA mRNA. In samples from $\mathrm{O}_{\Pi} \mathrm{s}$ injected with mRNAs 2 and 4, note the presence, in addition to the elongated RNA, of a species of slightly smaller size than the original transcript; this probably corresponds to an intermediate in the degradation of the mRNA that follows its elongation (see Fig. 2A; see also Figs. 9 and 10 in Huarte et al. 1987b).

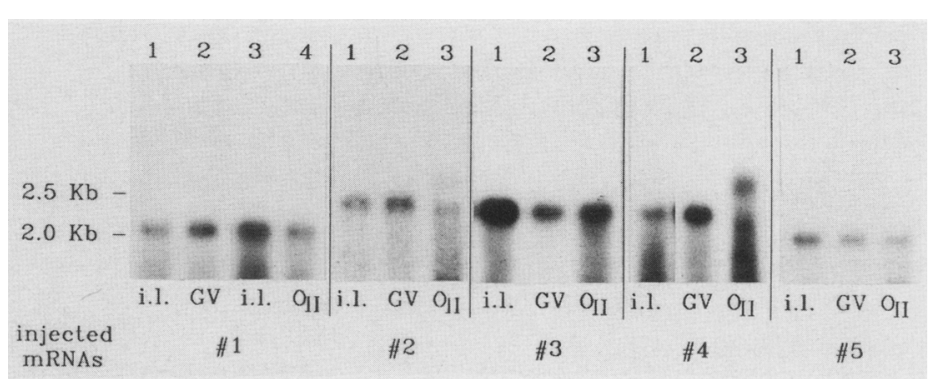


ment of tPA mRNA (Fig. 2), these two transcripts were elongated during meiotic maturation. In contrast, the size of transcripts 1,3 , and 5 did not change detectably. The low levels of uPA produced in oocytes injected with chimeric transcripts 3 and 5 suggest that nonpolyadenylated transcripts can be translated, although inefficiently. Alternatively, low levels of polyadenylated transcripts undetectable by Northern analysis may be generated by use of inefficient 3 '-processing signals, e.g., AAUAAU and AAUAAC sequences /Wickens and Stephenson 1984), which are present near the $3^{\prime}$ end of transcript 3.

Taken together, these results show that chimeric mRNAs containing part of the $3^{\prime} U T R$ of tPA mRNA, including an AAUAAA sequence, are modified and translated in maturing, but not in arrested, oocytes in a manner similar to the endogenous tPA mRNA. They are also compatible with the idea that $3^{\prime}$ polyadenylation is directly related to translational activation.

\section{Polyadenylation is necessary for translational activation}

Polyadenylation of endogenous and injected mRNAs occurs only on those mRNAs that can be activated or on RNA fragments that correspond to the $3^{\prime}$ end of activatable mRNAs. Polyadenylation is not a consequence of translation: (1) It can be detected $3 \mathrm{hr}$ after GVBD (Huarte et al. 1987b), that is, before the protein products become detectable [4-5 hr after GVBD (Huarte et al. 1985 ) blocked $3 \mathrm{hr}$ after GVBD by inhibitors of translation (Huarte et al. 1987b). To determine whether polyadenylation is necessary for translational activation, a uPA/ tPA chimeric mRNA was modified through incorporation of a $3^{\prime}$-terminal cordycepin $\left(3^{\prime}\right.$-deoxy-A) residue to prevent $3^{\prime}$ elongation (Fig. 1B, transcript 6 ). As expected, this mRNA was not elongated during meiotic maturation (Fig. 6A). Zymographic analysis of oocyte extracts revealed that the $3^{\prime}$-blocked mRNA remained untranslated in maturing mouse oocytes (Fig. 6B). In contrast, control and 3 '-blocked mRNAs were translated with similar efficiency after injection in $X$. laevis oocytes (Fig. 6C) or in a wheat germ lysate (Fig. 6D). Thus, a free $3^{\prime} \mathrm{OH}$ is required for both polyadenylation and translational activation of an injected chimeric mRNA. We conclude that polyadenylation is a prerequisite to translation of this chimeric mRNA in maturing mouse oocytes.

\section{Polyadenylated mRNAs are translated}

The dormant state of endogenous tPA mRNA and of all the injected mRNAs in primary oocytes may be a result of the absence of a poly(A) sequence of sufficient length. A polyadenylated message thus may be translated in arrested primary oocytes. To test this possibility, oocytes were injected with a uPA reporter mRNA that had been polyadenylated (200-400 As) in vitro (Fig. 1B, transcript 7). With this mRNA, both arrested primary oocytes and maturing oocytes synthesized similar amounts of uPA
(Fig. 7A, lanes 3 and 4); as expected, only matured oocytes contained tPA. No translation product was detected in oocytes that had been injected with the poly $(\mathrm{A})^{-}$transcripts (Fig. 7A, lanes 1 and 2; see also Fig. 4, lanes 1 and 2), although the translational efficiency of these poly $(\mathrm{A})^{+}$and poly $(\mathrm{A})^{-}$mRNAs in other systems (wheat germ lysate; Fig. 7B) was comparable. Similar results were also obtained with chimeric (uPA/tPA) mRNAs (Fig. 8): In all cases, an in vitro-added poly(A) sequence allowed efficient translation in primary oocytes. Thus, primary and secondary oocytes are equally competent to translate injected mRNAs, provided they contain a poly(A) tail of sufficient length. Therefore, the dormancy of the endogenous tPA mRNA appears to be the result of the lack of a long poly(A) tract, and regulated polyadenylation provides a mechanism for translational control.

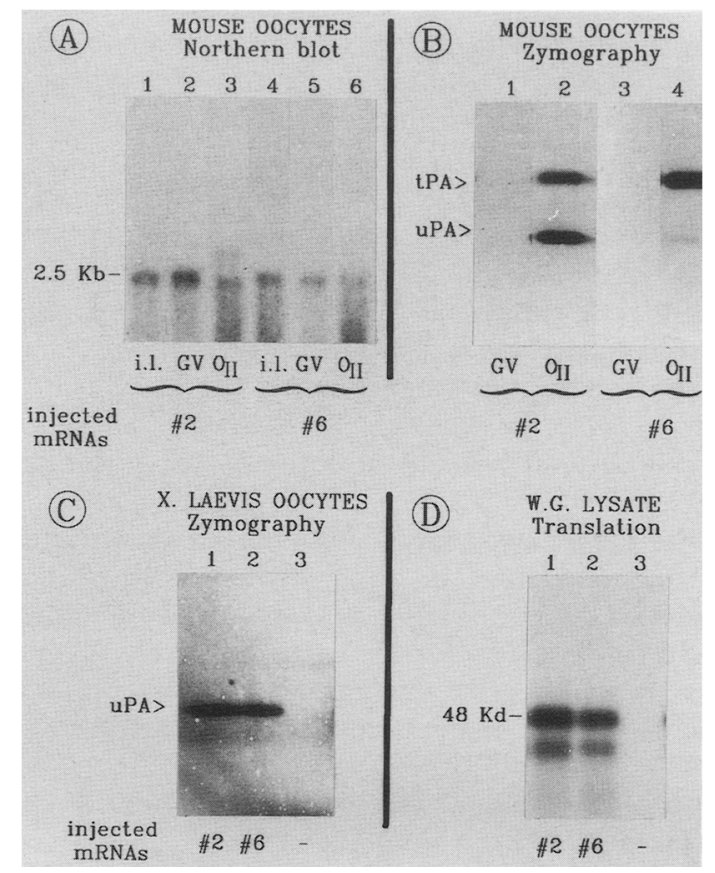

Figure 6. Injection of $3^{\prime}$-blocked chimeric mRNA. (A) Oocytes were injected with transcripts $B(2)$ and $B(6)$ (Fig. 1B) and lysed either immediately (i.l.) or after culture. RNA extraction and analysis by Northern blot hybridization was as for Fig. 5. $(B)$ Oocytes injected with the same transcripts as for $A$ were cultured, collected, and assayed by zymography, as for Fig. 4 (lanes 1-10). Four other preparations of these transcripts were tested in separate experiments, and three to five pools of oocytes were analyzed for each transcript preparation. $(C)$ The same transcripts as for $A$ were injected into stage VI $X$. laevis oocytes, and the oocytes were cultured, lysed, and assayed by zymography as for Fig. 4 (lanes 11-16). Oocytes were also injected with buffer alone (-, lane 3). (D) The same transcripts as for $A$ were added to a wheat germ translation extract in the presence of [ ${ }^{35}$ S]methionine. (-, lane 3) No mRNA added. The samples were analyzed by SDS-PAGE followed by fluorography. The $48-\mathrm{kD}$ band is $\mathrm{uPA}_{\text {; }}$ the lower $(45-\mathrm{kD})$ species results from the translation of an incomplete uPA mRNA (size $1.2 \mathrm{~kb}$, generated by pausing of the SP6 RNA polymerase), lacks the carboxy-terminal region of the protein and is enzymatically inactive. 


\section{Discussion}

We have shown that mRNAs injected into the cytoplasm of primary mouse oocytes can be subject to the same post-transcriptional processing and translational activation that control expression of endogenous mRNAs. The similar behavior of injected and endogenous RNAs observed in our experiments is reminiscent of the situation in $X$. laevis oocytes, in which injected $\mathrm{Vgl} \mathrm{mRNA}$ is transported to the vegetal cortex as is the endogenous transcript (Yisraeli and Melton 1988). Our results exclude certain mechanisms of translational control, in that they show that the information sufficient to designate an mRNA as dormant is present in the in vitro-synthesized transcript; they also suggest that timely mRNA activation in maturing oocytes relies, in part, on structural determinants present in the mRNAs themselves.

Two lines of evidence indicate that the $3^{\prime}$ UTR of tPA mRNA is essential for its translational activation in maturing oocytes. First, antisense-mediated amputation of this region prevents mRNA activation (Strickland et al. 1988); second, injected chimeric mRNAs containing this region are subject to a similar translational control as endogenous tPA mRNA. Two determinants of this 3'UTR are involved: one that allows the selective activation of endogenous tPA and chimeric uPA/tPA mRNAs, and the AAUAAA sequence that is known to function as a cleavage and polyadenylation signal in the processing of nuclear transcripts (Proudfoot and Brownlee 1976; Wickens and Stephenson 1984). The role of the 3'UTR appears to be primarily in targeting the mRNA for recognition by a cytoplasmic polyadenylation process: Polyadenylated mRNAs do not require this region for translation in arrested or maturing oocytes. These results are consistent with those obtained by Brinster and colleagues, who found that injected poly $(\mathrm{A})^{+}$mRNAs are translated with comparable efficiency in growing oocytes and fertilized eggs (Brinster et al. 1980; Ebert et al. 1984).

Polyadenylation is clearly a determining event in translational activation of tPA mRNA and of chimeric transcripts containing its $3^{\prime}$ UTR. We have shown previously that polyadenylation precedes accumulation of the protein product (Huarte et al. 1985, 1987b); in this present report we provide the two critical pieces of experimental evidence to show that poly(A) operates as a classical cis-acting element that is necessary and sufficient for translation: (1) The absence of poly(A) renders an mRNA silent, in either primary or maturing oocytes; (2) in vitro polyadenylation of a transcript allows its translation even in primary oocytes. It is important to note that endogenous tPA mRNA in primary oocytes already contains a short poly(A) segment (Huarte et al. $1987 \mathrm{~b})$. This segment, which is absent in tPA genomic DNA (R.J. Rickles, unpubl.) and, hence, added post-transcriptionally, is not sufficient for translation. Therefore, translational control must involve size discrimination of the poly(A) tract. What, then, is the mechanism of the extensive polyadenylation that occurs following resumption of meiosis? The requirement for an AAUAAA

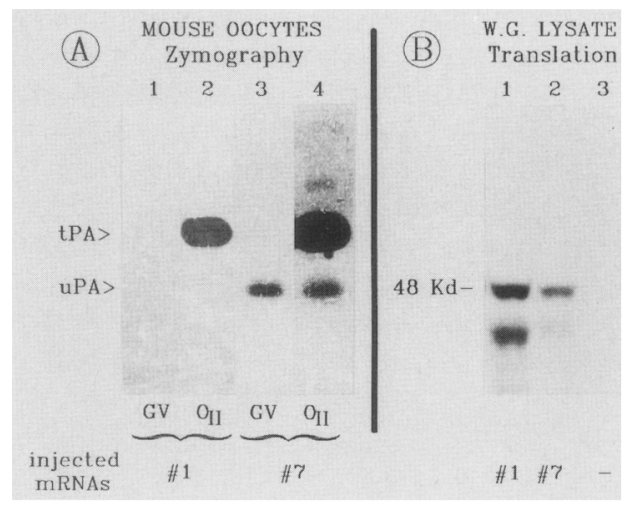

Figure 7. Translation of injected poly $(\mathrm{A})^{+}$mRNA. $(A)$ Oocytes were injected with transcripts $B(1)$ and $B(7)$ (Fig. $1 B)$, and cultured, collected, and assayed by zymography as in Fig. 4 flanes $1-10$ ). Three additional preparations of these mRNAs were tested in separate experiments; a total of at least 12 pools of GV oocytes and 19 pools of maturing $\left(\mathrm{BD}\right.$ or $\mathrm{O}_{\mathrm{II}}$ ) oocytes was analyzed for each of the two mRNAs. $(B)$ The same transcripts as for $A$ were translated in vitro in a wheat germ extract, as for Fig. 6D.

sequence close to the $3^{\prime}$ end of the mRNA suggests that the cytoplasmic adenylation process has a specificity comparable to that implicated in nuclear polyadenylation. An AAUAAA-dependent polyadenylation system that can act on precleaved mRNA precursors has recently been identified in nuclear extracts of HeLa cells (Zarkower et al. 1986; Christofori and Keller 1988; Takagaki et al. 1988). Whether such a nuclear polymerase is released into the cytoplasm upon breakdown of the germinal vesicle or whether a distinct enzyme is involved, is not known. Whatever the case may be, polyadenylation of tPA mRNA requires active protein synthesis during the first hours after GVBD (Huarte et al. $1987 \mathrm{~b}$ ), indicating that synthesis of a new protein is necessary, which could be a poly(A) polymerase or another protein involved in recognition of the mRNA by the polymerase.

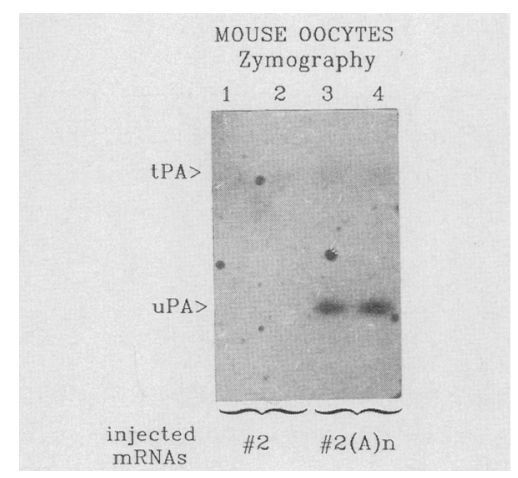

Figure 8. Translation of injected poly $(A)^{+}$mRNA. Oocytes were injected with transcript $B(2)(2$, lanes 1 and 2$)$ or transcript $B(2)$ that had been polyadenylated in vitro [2(A)n, lanes 3 and 4]. After culture for $10-12 \mathrm{hr}$ in the presence of dBcAMP, GV oocytes were collected and assayed by zymography, as in Fig. 4 (lanes 1-10). Duplicate pools of three oocytes were assayed in lanes 1 and 2 and in lanes 3 and 4 . 
How does polyadenylation of endogenous and injected mRNAs enhance their translation? This is not a result of increased stability of the polyadenylated transcripts, because RNAs that could not be polyadenylated (and therefore that remained nontranslated) were at least as stable as their poly $\left.(\mathrm{A})^{+}\right]$counterparts. Also, because poly $(\mathrm{A})^{+}$and $\operatorname{poly}(\mathrm{A})^{-}$mRNAs were equally well translated in two other systems, poly(A)-dependent translation in the mouse oocyte does not reflect a general difference in their translatability. At this time we can only speculate that the poly(A) tract interacts with a determinant that favors translation. This determinant appears to be already present in the arrested primary oocyte, or it may be a part of the mRNA [translation of poly $(\mathrm{A})^{+}$mRNA in primary oocytes]; the interaction requires a poly $(\mathrm{A})$ tract of sufficient length $\left(3^{\prime}\right.$ elongation during meiotic maturation). This model is supported by the observation that determinants that bind to long poly(A) tracts $(>30 \mathrm{As})$ enhance translation of poly $(\mathrm{A})^{+}$ mRNAs in reticulocyte lysates (Jacobson and Favreau 1983).

A recent paper by McGrew et al. (1989), using Xenopus oocytes, has many similarities with the present work. Both papers show that polyadenylation during meiotic maturation is dependent on mRNA-specific sequences and AAUAAA, and that it is necessary for translational recruitment. However, the final conclusions of the two papers are different. McGrew et al. found that a chimeric transcript polyadenylated in vitro was not present on polysomes after injection into immature Xenopus oocytes; they concluded that a long poly(A) tail is not sufficient for translation and that a dynamic process of polyadenylation is required. In our case, a polyadenylated reporter mRNA was translated in primary mouse oocytes (Figs. 7 and 8), directly showing that the presence of a long poly(A) tail is sufficient. These contrasting results might be explained by differences between Xenopus and mouse oocytes. An alternative explanation could be the use of different assay systems. McGrew et al. used the presence of their transcripts on polysomes at a discrete time point $(5-8 \mathrm{hr}$ after injection) as a measure of translational recruitment. In our case, we measured the accumulated translation product, which provides an integrated signal over the entire period of incubation and may better reveal transient activation. Despite these differences, the similarities in the two systems emphasize the likely generality of regulated polyadenylation as a basic mechanism for translational activation of maternal mRNAs.

In somatic cells, the importance of the translational control mechanism described here cannot be evaluated unambiguously on the basis of available data. However, it is striking that increased lengths of poly(A) on the relevant mRNAs have been correlated with enhanced synthesis of vasopressin (Carrazana et al. 1988; Robinson et al. 1988), insulin (Muschel et al. 1986), and growth hormone (Paek and Axel 1987); it is not known whether this corresponds to de novo nuclear synthesis of longer transcripts, to enhanced stability of the poly(A) tail, or to cytoplasmic poly(A) addition onto pre-existing mRNAs.
In any event, our data, which identify an in vivo role for the $3^{\prime}$-poly(A) tract, should focus attention on the regulation of this post-transcriptional modification (Palatnik et al. 1984; Galili et al. 1988) in the context of translational control.

\section{Methods \\ Mouse oocyte collection and culture}

Procedures for oocyte collection from B6/D2 $F_{1}$ hybrid mice, culture, and injection were as described previously (Huarte et al. 1985, 1987b; Strickland et al. 1988). In all experiments, $\sim 10$ pl was injected into the cytoplasm of primary oocytes. Oocytes were cultured in the presence of dibutyryl cAMP $(100 \mu \mathrm{g} / \mathrm{ml})$, to prevent resumption of meiosis, or in the absence of dBcAMP, to allow meiotic maturation.

\section{Plasmid constructions}

The numbering corresponds to the transcripts represented schematically in Figure 1.

A(1)-(2) A DraI fragment containing the 455 nucleotides, 3' terminal of mouse tPA cDNA (positions 2050-2504; Rickles et al. 1988), was cloned in the indicated orientation into the HincII site of pSP64 (Melton et al. 1984). Transcription templates were generated by linearizing this plasmid with: for $\mathrm{A}(1)$, $\mathrm{XbaI}$ - the construct yields a 487-nucleotide transcript, with a 3 '-terminal G-and for A(2), SpeI-it yields a 380-nucleotide transcript, $3^{\prime}$-terminal $\mathrm{G}$, lacking the 99 nucleotides, $3^{\prime}$-terminal of the 3'UTR of tPA cDNA. Other templates were prepared from this plasmid, using different restriction enzymes to yield transcripts (not illustrated in Fig. 1) with different nucleotides at their $3^{\prime}$ ends (for details, see Fig. 3).

A(3) A 155-nucleotide BgIII-DraI fragment containing the entire $3^{\prime}$ UTR and 63 nucleotides of $3^{\prime}$-flanking sequences of a rabbit $\beta$-globin gene (positions 1196-1351; Rohrbauch et al. 1985 ; kindly provided by N. Proudfoot, Oxford University) was subcloned between the BamHI and SmaI sites of pSP64. The plasmid was linearized with NdeI to yield a 165-nucleotide transcript.

A(4) A 366-nucleotide BgIII-SmaI fragment containing part of the 3'UTR of mouse UPA cDNA and a synthetic AATAAA element [see B $(5)$, below], was subcloned between the BamHI and SmaI sites of pSP64. The plasmid was linearized with SmaI to yield a 399-nucleotide transcript containing an AAUAAA element.

$B$ (1) An XbaI-SmaI fragment, containing 22 nucleotides of the 5'UTR, the entire coding sequence, and 679 nucleotides of the 3'UTR of mouse uPA cDNA (positions 37-2036; Belin et al. 1985), was cloned between the cognate sites of pSP64; this construct lacks the 277 nucleotides, $3^{\prime}$ terminal of the $3^{\prime}$ UTR of UPA cDNA.

$B(2)$ The plasmid containing the 455 nucleotides, $3^{\prime}$-terminal of mouse tPA cDNA (see A(1)-(2) above), was linearized with HindIII, the ends were filled in with the Klenow fragment of DNA polymerase I, and the HincII-SmaI fragment of construct $\mathrm{B}(1)$ containing uPA sequences was inserted.

$B(3)-(4)$ Construct $B(2)$ was digested with SpeI and BamHI, the ends were filled in, and the large fragment was isolated (this 
digestion removes the 99 nucleotides, 3' terminal of tPA-derived sequences). A 20-bp synthetic fragment containing an AATAAA element [GATCCAATAAAGCTCGAATT, derived from pUCCATpA (Linney and Donerly 1983) by BamHI-EcoRI digestion and filling in] was inserted; the orientation of the insert was verified by plasmid sequencing. This construct, when linearized with BamHI, yields a transcript lacking AAUAAA $[\mathrm{B}(3)]$; when linearized with $S m a \mathrm{I}$, it yields a transcript containing AAUAAA [B(4)].

$B(5)$ The AATAAA element isolated from construct $B(3)-(4)$ as a $27-\mathrm{bp} B a m \mathrm{HI}-\mathrm{SmaI}$ fragment was cloned into the cognate sites of pSP64. This plasmid was linearized with HindIII, filled in, and ligated to the filled-in XbaI-SmaI fragment of the uPA cDNA [construct $\mathrm{B}(1)]$.

\section{Preparation of transcripts}

Transcripts were prepared with SP6 RNA polymerase, as described (Huarte et al. 1987b; Strickland et al. 1988), and dissolved at $100 \mathrm{ng} / \mu \mathrm{l}$ in $150 \mathrm{mM} \mathrm{KCl}$. Transcript $\mathrm{B}(6)$ : transcript $\mathrm{B}(2)(10 \mu \mathrm{g} / \mathrm{ml})$ was incubated for $30 \mathrm{~min}$ at $37^{\circ} \mathrm{C}$ with Escherichia coli poly(A) polymerase $(B R L, 30 \mathrm{U} / \mathrm{ml})$ in buffer $\mathrm{A}$ [50 mM Tris-Cl (pH 8.1), $250 \mathrm{~mm} \mathrm{NaCl}, 10 \mathrm{~mm} \mathrm{MgCl}_{2}, 2 \mathrm{~mm}$ DTT, $100 \mu \mathrm{g} / \mathrm{ml}$ BSA, $1000 \mathrm{U} / \mathrm{ml}$ RNasin (Promega)], containing 100 $\mu \mathrm{M}$ cordycepin 5 '-triphosphate (Pharmacia). To remove molecules that may not have incorporated cordycepin, the transcript was polyadenylated (same conditions, except $100 \mu \mathrm{M}$ ATP instead of cordycepin), purified, dissolved in water, and adsorbed onto poly(U)-Sepharose (Huarte et al. 1987b); 3'-blocked transcript was purified from the unbound material. Transcript $B(7)$ was prepared from transcript $B(1)$, and transcript $2(A)$ n from transcript $\mathrm{B}(2)$, by incubation with $E$. coli poly $(\mathrm{A})$ polymerase in buffer A containing $100 \mu \mathrm{M} \mathrm{ATP}$, as described for transcript $\mathrm{B}(6)$.

To prepare radiolabeled transcripts, the specific activity of [32P]UTP was raised to $4 \mu \mathrm{Ci} / \mu \mathrm{l}$ and the total UTP concentration was $100 \mu \mathrm{M}$; the final concentration of these RNAs was $300 \mathrm{ng} / \mu \mathrm{l}$.

\section{RNA analysis}

RNA extraction and Northern blot hybridizations were performed as described (Huarte et al. 1987b), using probes transcribed from pSP64-MT $\mathrm{MT}_{3}$ (Huarte et al. 1987b) [complementary to positions 1680-2405 of tPA mRNA (Rickles et al. 1988) (Figs. 2 and 3)], or from pDB4501 [complementary to positions 420-1078 of uPA mRNA (Belin et al. 1985; Huarte et al. 1987a) (Figs. 5 and 6)]. Gel analysis of radiolabeled transcripts was performed by electrophoresis in $6 \%$ polyacrylamide/urea gels, and autoradiography of the dried gels. RNase $\mathrm{H} \pm$ oligo(dT) treatment was as described (Huarte et al. 1987b).

\section{Translation assays}

Mouse oocytes were collected in groups of three after culture, lysed in Triton X-100, and assayed by zymography (Huarte et al. 1985). Stage VI $X$. laevis oocytes (10 oocytes per group) were injected with transcripts diluted to $10 \mathrm{ng} / \mu \mathrm{l}$, or with buffer alone, collected following $20 \mathrm{hr}$ of culture, and analyzed by zymography (Belin et al. 1984). For in vitro translation, transcripts were added $(1 \mathrm{ng} / \mu \mathrm{l})$ to a wheat germ translation extract [prepared as described (Dobberstein and Blobel 1977) and used at $35 \%$ final concentration] in the presence of $\left[{ }^{35} \mathrm{~S}\right]$ methionine; the samples were analyzed by SDS-PAGE, followed by fluorography.

\section{Acknowledgments}

We thank J.-P. Gerber and T. Daraio for excellent artwork and photography. This work was supported by grants from the Fonds National Suisse de la Recherche Scientifique (3.0591.87), the Sandoz Stiftung zur Förderung der medizinisch-biologischen Wissenschaften, the National Institutes of Health (HD-17875 and HD-25922), the American Cancer Society (BC-525), and an American Cancer Society Scholar grant to S.S (SG-145) Roma.

\section{References}

Belin, D., F. Godeau, and J.-D. Vassalli. 1984. Tumor promoter PMA stimulates the synthesis and secretion of mouse prourokinase in MSV-transformed 3T3 cells: This is mediated by an increase in urokinase mRNA content. EMBO $J$. 3: 1901-1906.

Belin, D., J.-D. Vassalli, C. Combépine, F. Godeau, Y. Nagamine, E. Reich, H.P. Kocher, and R.M. Duvoisin. 1985. Cloning, nucleotide sequencing and expression of cDNAs encoding mouse urokinase-type plasminogen activator. Eur. J. Biochem. 148: 225-232.

Brawerman, G. 1981. The role of the poly(A) sequence in mammalian messenger RNA. CRC Crit. Rev. Biochem. 10: 1-38.

Brinster. R.L., H.Y. Chen, M.E. Trumbauer, and M.R. Avarbock. 1980. Translation of globin messenger RNA by the mouse ovum. Nature 283: 499-501.

Carrazana, E.J., K.B. Pasieka, and J.A. Majzoub. 1988. The vasopressin mRNA poly(A) tract is unusually long and increases during stimulation of vasopressin gene expression in vivo. Mol. Cell. Biol. 8: 2267-2274.

Christofori, G. and W. Keller. 1988. Poly(A) polymerase purified from HeLa cell nuclear extract is required for both cleavage and polyadenylation of pre-mRNA in vitro. Cell 54: 875879.

Colot, H.V. and M. Rosbash. 1982. Behaviour of individual maternal poly $(\mathrm{A})^{+}$RNAs during embryogenesis of Xenopus laevis. Dev. Biol. 94: 79-86.

Darnell, J.E., R. Wall, and R.J. Tushinski. 1971. An adenylic acid-rich sequence in messenger RNA of HeLa cells and its possible relationship to reiterated sites in DNA. Proc. Natl. Acad. Sci. 68: 1321-1325.

Davidson, E.H. 1986. Gene activity in early development, 3rd ed. Academic Press, New York.

Dobberstein, B. and G. Blobel. 1977. Functional interaction of plant ribosomes with animal microsomal membranes. Biochem. Biophys. Res. Commun. 74: 1675-1682.

Doel, M.T. and N.H. Carey. 1976. The translational capacity of deadenylated ovalbumin messenger RNA. Cell 8: 51-58.

Drummond, D.R., J. Armstrong, and A. Colman. 1985. The effect of capping and polyadenylation on the stability, movement and translation of synthetic RNAs in Xenopus oocytes. Nucleic Acids Res. 13: 7375-7394.

Dworkin, M.B. and E. Dworkin-Rastl. 1985. Changes in RNA titers and polyadenylation during oogenesis and oocyte maturation in Xenopus laevis. Dev. Biol. 112: 451-457.

Ebert, K.M., B.V. Paynton, G.S. McKnight, and R.L. Brinster. 1984. Translation and stability of ovalbumin messenger RNA injected into growing and fertilized ova of mice. J. Embryol. Exp. Morphol. 84: 91-103.

Edmonds, M. and M.G. Caramela. 1969. The isolation and characterization of adenosine monophosphate-rich polynucleotides synthesized by Ehrlich ascites cells. J. Biol. Chem. 244: 1314-1324.

Edmonds, M., M.H. Vaughan Jr., and H. Nakazato. 1971. Polyadenylic acid sequences in the heterogeneous nuclear RNA 
and rapidly-labeled polyribosomal RNA of HeLa cells: Possible evidence for a precursor relationship. Proc. Natl. Acad. Sci. 68: 1336-1340.

Galili, G., E.E. Kawata, L.D. Smith, and B.A. Larkins. 1988. Role of the 3 '-poly(A) sequence in translational regulation of mRNAs in Xenopus laevis oocytes. J. Biol. Chem. 263: $5764-5770$.

Goldman, D.S., A.A. Kiessling, and G.M. Cooper. 1988. Posttranscriptional processing suggests that $\mathrm{c}-\mathrm{mos}$ functions as a maternal message in mouse eggs. Oncogene 3: 159-162.

Huarte, J., D. Belin, and J.-D. Vassalli. 1985. Plasminogen activator in mouse and rat oocytes: Induction during meiotic maturation. Cell 43: 551-558.

Huarte, J., D. Belin, D. Bosco, A.-P. Sappino, and J.-D. Vassalli. 1987a. Plasminogen activator and mouse spermatozoa: Urokinase synthesis in the male genital tract and binding of the enzyme to the sperm cell surface. $J$. Cell Biol. 104: 12811289.

Huarte, J., D. Belin, A. Vassalli, S. Strickland, and J.-D. Vassalli. 1987b. Meiotic maturation of mouse oocytes triggers the translation and polyadenylation of dormant tissue-type plasminogen activator mRNA. Genes Dev. 1: 1201-1211.

Huez, G., G. Marbaix, E. Hubert, M. Leclercq, U. Nudel, H. Soreq, R. Salomon, B. Lebleu, M. Revel, and U.Z. Littauer. 1974. Role of the polyadenylate segment in the translation of globin messenger RNA in Xenopus oocytes. Proc. Natl. Acad. Sci. 71: 3143-3146.

Hyman, L.E. and W.M. Wormington. 1988. Translational inactivation of ribosomal protein mRNAs during Xenopus oocyte maturation. Genes Dev. 2: 598-605.

Ilan, J., ed. 1987. Translational regulation of gene expression. Plenum Press, New York.

Jacobson, A. and M. Favreau. 1983. Possible involvement of poly(A) in protein synthesis. Nucleic Acids Res. 11: 63536368.

Lee, S.Y., J. Mendecki, and G. Brawerman. 1971. A polynucleotide segment rich in adenylic acid in the rapidly-labeled polyribosomal RNA component of mouse sarcoma 180 ascites cells. Proc. Natl. Acad. Sci. 68: 1331-1335.

Linney, E. and S. Donerly. 1983. DNA fragments from F9 PyEC mutants increase expression of heterologous genes in transfected F9 cells. Cell 35: 693-699.

McGrew, L.L., E. Dworkin-Rastl, M.B. Dworkin, and J.D. Richter. 1989. Poly(A) elongation during Xenopus oocyte maturation is required for translational recruitment and is mediated by a short sequence element. Genes Dev. 3: 803815.

Melton, D.A., P.A. Krieg, M.R. Rebagliati, T. Maniatis, K. Zinn, and M.R. Green. 1984. Efficient in vitro synthesis of biologically active RNA and RNA hybridization probes from plasmids containing a bacteriophage SP6 promoter. Nucleic Acids Res. 12: 7035-7056.

Müllner, E.W. and L.C. Kühn. 1988. A stem-loop in the 3' untranslated region mediates iron-dependant regulation of transferrin receptor mRNA stability in the cytoplasm. Cell 53: 815-825.

Muschel, R., G. Khoury, and L.M. Reid. 1986. Regulation of insulin mRNA abundance and adenylation: Dependence on hormones and matrix substrata. Mol. Cell. Biol. 6: 337-341.

Mutter, G.L., G.S. Grills, and D.J. Wohlgemuth. 1988. Evidence for the involvment of the proto-oncogene c-mos in mammalian meiotic maturation and possibly very early embryogenesis. EMBO J. 7: 683-689.

Paek, I. and R. Axel. 1987. Glucocorticoids enhance stability of human growth hormone mRNA. Mol. Cell. Biol. 7: 14961507.
Palatnik, C.M., C. Wilkins, and A. Jacobson. 1984. Translational control during early Dictyostelium development: Possible involvment of poly(A) sequences. Cell 36: 10171025.

Paynton, B.V., R. Rempel, and R. Bachvarova. 1988. Changes in state of adenylation and time course of degradation of maternal mRNAs during oocyte maturation and early embryonic development in the mouse. Dev. Biol. 129: 304-314.

Proudfoot, N.J. and G.G. Brownlee. 1976. 3' non-coding region sequences in eukaryotic messenger RNA. Nature 263: $211-$ 214.

Rickles, R.J., A.L. Darrow, and S. Strickland. 1988. Molecular cloning of complementary DNA to mouse tissue plasminogen activator mRNA and its expression during F9 teratocarcinoma cell differentiation. I. Biol. Chem. 263: 15631569.

Robinson, B.G., D.M. Frim, W.J. Schwartz, and J.A. Majzoub. 1988. Vasopressin mRNA in the suprachiasmatic nuclei: Daily regulation of polyadenylate tail length. Science 241: $342-344$

Rohrbauch, M.L., J.E. Johnson, M.D. James, and R.C. Hardison. 1985. Transcription unit of the rabbit $\beta 1$ globin gene. Mol. Cell. Biol. 5: 147-160.

Rosenthal, E.T. and J.V. Ruderman. 1987. Widespread changes in the translation and adenylation of maternal messenger RNAs following fertilization of Spisula oocytes. Dev. Biol. 121: $237-246$

Rosenthal, E.T., T.R. Tansey, and J.V. Ruderman. 1983. Sequence-specific adenylations and deadenylations accompany changes in the translation of maternal mRNA after fertilization of Spisula oocytes. I. Mol. Biol. 166: 309-327.

Shull, G.E. and E.C. Theil. 1982. Translational control of ferritin synthesis by iron in embryonic reticulocytes of the bullfrog. J. Biol. Chem. 257: 14187-14191.

Strickland, S., J. Huarte, D. Belin, A. Vassalli, R.J. Rickles, and J.-D. Vassalli. 1988. Antisense RNA directed against the 3' noncoding region prevents dormant mRNA activation in mouse oocytes. Science 241: 680-684.

Takagaki, Y., L.C. Ryner, and J.L. Manley. 1988. Separation and characterization of a poly(A) polymerase and a cleavage/ specificity factor required for pre-mRNA polyadenylation. Cell 52: 731-742.

Wickens, M. and P. Stephenson. 1984. Role of the conserved AAUAAA sequence: Four AAUAAA point mutants prevent messenger RNA 3' end formation. Science 226: 1045-1051.

Yen, T.J., D.A. Gay, J.S. Pachter, and D.W. Cleveland. 1988. Autoregulated changes in the stability of polyribosomebound $\beta$-tubulin mRNAs are specified by the first 13 translated nucleotides. Mol. Cell. Biol. 8: 1224-1235.

Yisraeli, J.K. and D.A. Melton. 1988. The maternal mRNA Vg1 is correctly localized following injection into Xenopus oocytes. Nature 336: 592-595.

Zähringer, J., B.S. Baliga, and H.N. Munro. 1976. Novel mechanism for translational control in regulation of ferritin synthesis by iron. Proc. Natl. Acad. Sci. 73: 857-861.

Zarkower, D., P. Stephenson, M. Sheets, and M. Wickens. 1986. The AAUAAA sequence is required both for cleavage and for polyadenylation of simian virus 40 pre-mRNA in vitro. Mol. Cell. Biol. 6: 2317-2323. 


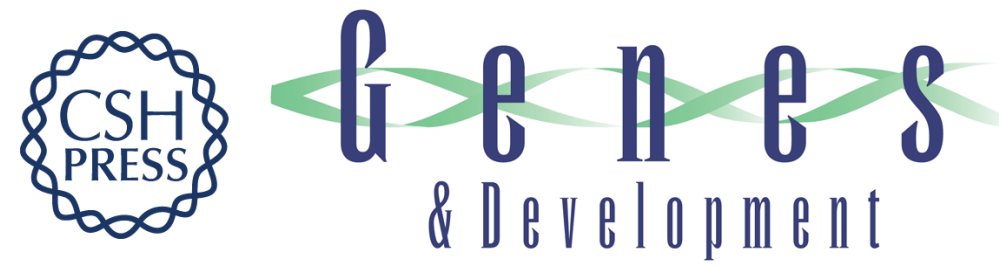

\section{Regulated polyadenylation controls mRNA translation during meiotic maturation of mouse oocytes.}

J D Vassalli, J Huarte, D Belin, et al.

Genes Dev. 1989, 3:

Access the most recent version at doi:10.1101/gad.3.12b.2163

References This article cites 47 articles, 22 of which can be accessed free at:

http://genesdev.cshlp.org/content/3/12b/2163.full.html\#ref-list-1

License

Email Alerting

Service

Receive free email alerts when new articles cite this article - sign up in the box at the top right corner of the article or click here.

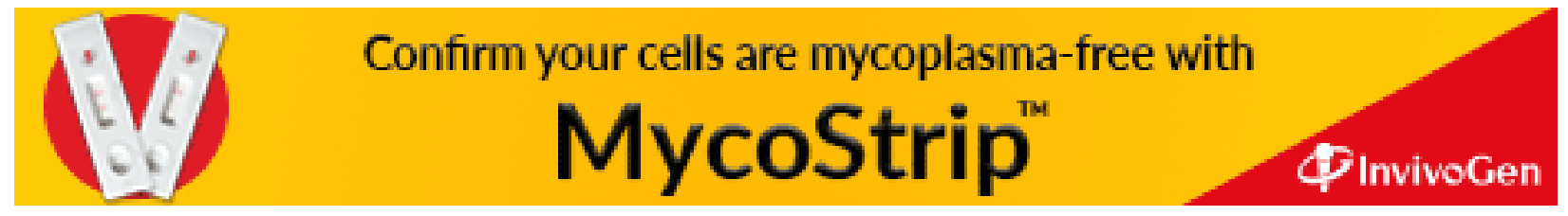

